Original Paper http://ajol.info/index.php/ijbcs http://indexmedicus.afro.who.int

\title{
Évaluation de quinze génotypes de niébé [Vigna unguiculata (L.) Walp.] Sous conditions pluviales dans le Nord et le Centre Ouest du Burkina Faso
}

\author{
Wendbenedo Joël Antoine LALSAGA ${ }^{1^{*}}$ et Issa DRABO ${ }^{2}$ \\ ${ }^{1}$ Université de Ouagadougou (UFR-SVT) 09 BP 848 Ouagadougou 09, Burkina Faso. \\ ${ }^{2}$ Département Oléo- Protéagineux, Institut de l'Environnement et de Recherches Agricoles Saria, $04 B P 8645$ \\ Ouagadougou, Burkina Faso. \\ ^Auteur correspondant ; E-mail : wjoellalsaga@gmail.com ; Tel : +22672485015
}

\section{RESUME}

Le niébé est une denrée de base en Afrique subsaharienne. L'objectif de ce travail est d'identifier des lignées à haut rendement, tolérantes à la sécheresse et résistantes à la race 1 de $S$. gesnerioides. Des lignées issues de la sélection récurrente et des variétés témoins ont été évaluées au Centre Ouest (condition maximale d'humidité) et au Nord (condition de stress hydrique) du Burkina Faso en 2014. Tous les génotypes ont montré une résistance à la race 1 de Striga et plusieurs lignées ont réussi à produire plus de $1300 \mathrm{~kg} / \mathrm{ha}$ sur les deux sites. L'Analyse en Composantes Principales réalisée sur la base des caractères quantitatifs a permis d'identifier deux groupes. Le groupe 1 est composé par des individus précoces à rendement modéré (Nafi, Gorom local, KVX-61-1 et Komcalle). Le groupe 2 est constitué par les génotypes extra précoces à rendement modéré (C2-6-3, C2-18-2, C2-27-2, IT97K-499-35 et Yiis yande).

(C) 2017 International Formulae Group. All rights reserved.

Mots clés: Niébé, Striga gesnerioides, stress hydrique, rendement.

\section{Evaluation of 15 genotypes of rain-fed cowpea [Vigna unguiculata (L.) Walp.] In northern and central-western Burkina Faso}

\begin{abstract}
Cowpea is a staple food in sub-Saharan Africa. The objective of this work is to identify high yielding, drought tolerant and race 1 resistant lines of $S$. gesnerioides. The breeding lines of including some varieties were evaluated in field trial under drought stressed and non-stressed conditions in 2014. Results show that the breeding lines showed complete resistance to race 1 of Striga and many improved varieties are capable of producing over $1300 \mathrm{~kg}$ grain/ha. Biplot displays indicated that the genotypes could be grouped into two categories according to their cycle and yielding ability as indicated below: moderate yielding-early maturing (Nafi, Gorom local, KVX-61-1 et Komcalle) and moderate yielding extra-early maturing (C2-6-3, C2-18-2, C2-27-2, IT97K-499-35 et Yiis yande).

(C) 2017 International Formulae Group. All rights reserved.
\end{abstract}

Keywords: Cowpea, Striga gesnerioides, drought, yield. 


\section{INTRODUCTION}

Le niébé (Vigna unguiculata (L.) Walp.) est une légumineuse très abondante dans les zones chaudes et semi arides de l'Afrique, où les autres cultures ont des difficultés pour pousser à cause des fortes températures, de la sécheresse et des sols peu fertiles (Hall, 2004). Au Sahel, où la saison pluvieuse est de courte durée (trois mois), le niébé est largement cultivé sur des sols sableux à faible capacité de rétention d'eau et est ainsi exposé à des déficits hydriques postfloraison qui entravent sa productivité (Fatokun et al., 2009). Malgré sa réputation de plante résistante à la sécheresse, les déficits hydriques récurrents réduisent significativement sa croissance et sa productivité (Muchero et al., 2008). Lorsque la sécheresse intervient en fin de saison pluvieuse, elle est plus dommageable car elle affecte la floraison et le remplissage des gousses, entraînant ainsi une baisse du rendement (Chuilele, 2010). Au Burkina Faso, la production de niébé est aussi affectée par des contraintes biotiques. La pression des ravageurs des cultures est assez importante pour la plupart des producteurs (Rabe et al., 2017). Les insectes, les parasites et les maladies de niébé sont plus abondants au cours des phases de croissance végétative, de floraison et de fructification-maturation (Issoufou et al., 2017). Le Striga gesnerioides est aussi un important facteur qui entraîne une baisse de la production de niébé. Les pertes de rendement que cause cette plante peuvent prendre des dimensions de véritables fléaux économiques. En Afrique de l'Ouest, la présence de $S$. gesnerioides a été notée au Burkina Faso, au Mali, au Togo, au Nigeria et au Benin, avec au moins une race dans chaque pays. Au moins trois races de cette plante parasite ont été répertoriées au Burkina Faso. Des variétés de niébé résistantes au $S$. gesnerioides, avec un cycle court pouvant échapper à la sécheresse de fin-de-cycle ont été mises au point par l'INERA, grâce à des techniques conventionnelles d'amélioration, la sélection de races locales performantes et à l'introduction de cultivars. Les génotypes
KVx771-10 et KVx775-33-2 sont résistants aux trois races de $S$. gesnerioides qui prévalent au Burkina et possèdent un cycle de semi maturité de 70 jours (Tignegré, 2010). Au Burkina Faso cependant, il existe très peu de variétés résistantes aux trois races de $S$. gesnerioides et adaptées à la sécheresse. L'objectif de ce travail est d'identifier des lignées à haut rendement, tolérantes à la sécheresse et résistantes à la race 1 de $S$. gesnerioides.

\section{MATERIEL ET METHODES}

L'étude a été conduite de juillet à septembre 2014 dans deux stations de l'INERA notamment au Centre Ouest à Saria (condition maximale d'humidité) et au Nord à Pobé Mengao (condition de stress) du Burkina Faso (Figure 1). Le climat de Saria est de type nord soudanien caractérisé par une longue saison sèche qui va d'octobre à mai et une saison des pluies de juin à septembre. La station agronomique de Saria dispose d'une station météorologique avec suivi quotidien de la pluie, ETP et la température de l'air. La pluviométrie moyenne annuelle est de 800 $\mathrm{mm}$, mais cette moyenne cache de grandes fluctuations annuelles. Le climat de Pobe est de type sahélien caractérisé par une longue saison sèche qui va d'octobre à juillet et une saison des pluies de juillet à septembre. La pluviométrie moyenne annuelle est comprise entre $300 \mathrm{~mm}$ et $600 \mathrm{~mm}$.

\section{Matériel végétal}

Le germoplasm de niébé utilisé a été constitué de quinze entrées dont sept lignées fixées obtenues à partir de la sélection récurrente et huit variétés témoins. Parmi les sept lignées fixées, il y a eu une lignée à graines blanches $(\mathrm{C} 2-9-1)$ et quatre lignées à graines brunes (C2-6-3, C2-11-3, C2-18-2 et C2-27-2). Les lignées C2-111-2 et C2-111-4 ont été utilisées comme des contrôles négatifs. Les variétés témoins ont été les deux parents $\mathrm{du}$ croisement (Gorom local et IT97K-49935). Niizwe et Yiis yande ont déjà été identifiées comme des variétés extra précoces, à cycle semi maturité de 60 jours. Tiligre, 
KVX-61-1 et Nafi sont des variétés précoces de 70 jours.

\section{Dispositif expérimental}

Le dispositif utilisé a été un bloc de Fisher avec 5 répétitions. Les quinze entrées ont été semées dans un champ infesté de Striga. Chaque parcelle élémentaire a été constituée de 4 lignes de $4 \mathrm{~m}$, semées avec un écartement de $80 \times 40 \mathrm{~cm}$ à deux graines par poquet. Les données collectées concernent $50 \%$ floraison, le taux de chlorophylle, l'émergence de Striga, 95\% maturité, le rendement et le poids de 100 graines. Le nombre de jours de 50\% floraison a été évalué sur la base d'une observation visuelle. Le nombre de jour de $95 \%$ maturité a été évalué sur la base d'une observation visuelle du péricarpe des plants de chaque parcelle élémentaire. Pour le rendement les gousses de chaque parcelle élémentaire ont été récoltées et après battage ont été pesées. Le rendement a été obtenu en ramenant la production de chaque parcelle élémentaire à l'hectare. Le poids de 100 graines a été obtenu en effectuant un comptage manuel de 100 graines de niébé de chaque lignée suivi de la prise de la masse. La teneur en chlorophylle totale a été évaluée au 45ème jour après semis puis une semaine après soit au 52ème jour après semi. Les variables qualitatives observées ont été le flétrissement, la chute des feuilles et la présence de Striga gesnerioides.

\section{Analyse des données}

Le logiciel Genstat v4.10.3 a été utilisé pour l'analyse de variance. Une ANOVA à un facteur a été faite pour évaluer les différences génotypiques pour les paramètres mesurés dans chaque régime hydrique. Le logiciel XLSTAT-pro version 7.1 a été utilisé pour l'analyse en composantes principales (ACP).

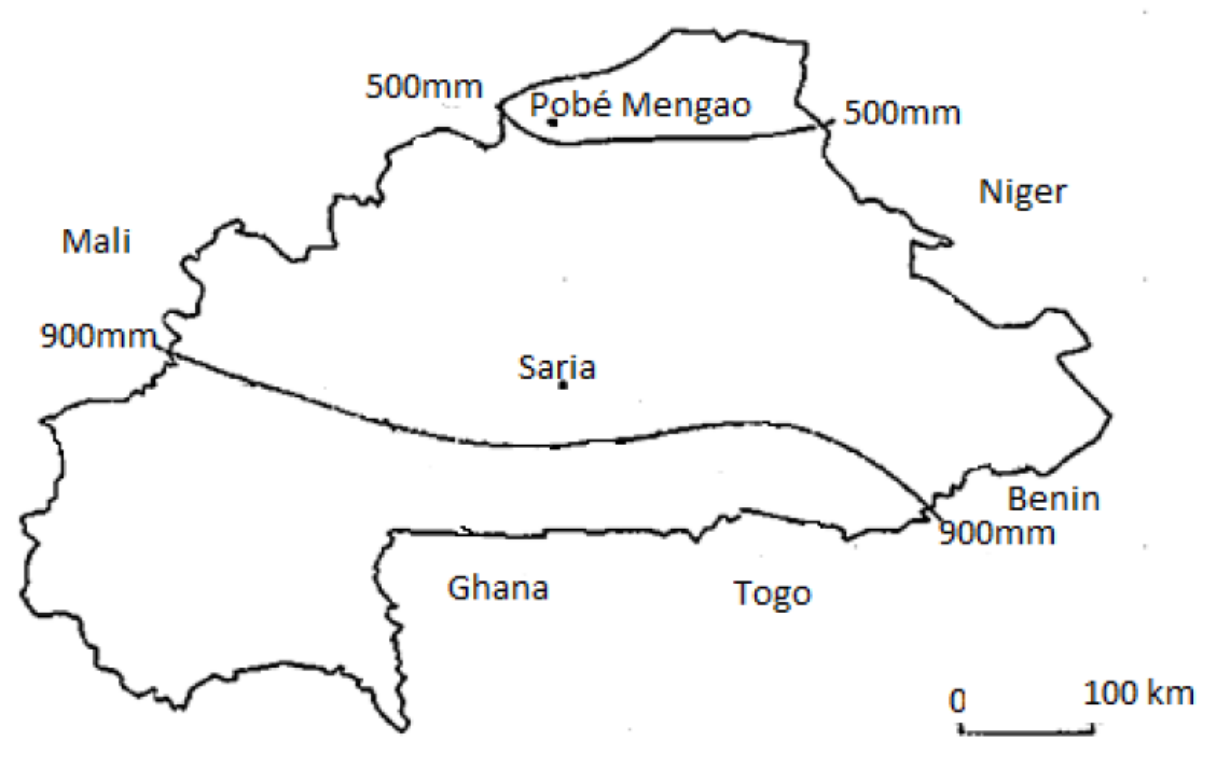

Cote d'Ivoire

Figure 1: Carte de localisation de Pobe Mengao et Saria. 


\section{RESULTATS}

Les résultats de l'évaluation des quinze génotypes (Tableau 1) ont montré une différence significative $(\mathrm{p}=0.001)$ entre les individus pour les dates de $50 \%$ floraison, 95\% maturité, le poids de 100 graines sur chacun des deux sites. Les plantes ont fleuri plus vite à Saria qui représente le lieu où il $\mathrm{y} a$ eu le maximum d'humidité. Le nombre de jours à $50 \%$ floraison a varié de 38 à 49 JAS à Pobé et 37 à 48 JAS pour le site de Saria. La variété Niizwe a été la première à fleurir sur les deux sites. Toutes les lignées ont fleuri avant le 45eme jour sauf C-92-1, C2-11-3 et C2-111-4. Le nombre moyen de jours à 95\% maturité pour les quinze génotypes a été 65,7 JAS à Pobé et 65,1 JAS à Saria. Au niveau des deux localités, toutes les lignées ont atteint la maturité avant 70 JAS. La variété Niizwe a été la première à atteindre $95 \%$ de maturité. Les génotypes KVX61-1 et Tiligre ont atteint respectivement 95\% maturité 70,8 JAS à Saria et 69 JAS à Pobé Mengao. Ils ont été les plus tardifs. Le rendement moyen pour tous les génotypes a été $1195,2 \mathrm{~kg} / \mathrm{ha}$ au niveau de la station de Saria et $1131,3 \mathrm{~kg} / \mathrm{ha}$ à Pobé. Le contrôle négatif C2-111-4 a donné le plus faible rendement au niveau des deux sites; il a produit moins de $500 \mathrm{~kg} / \mathrm{ha}$. Le parent Gorom local a produit $1421 \mathrm{~kg} / \mathrm{ha}$ à Saria et $1328 \mathrm{~kg} / \mathrm{ha}$ à Pobé. La lignée C2-9-1 a produit plus de $1500 \mathrm{~kg} / \mathrm{ha}$ à Saria. Le parent Gorom local a donné un poids de 100 graines de $16 \mathrm{~g}$ à Saria et $15 \mathrm{~g}$ à Pobé. Le parent IT97K-499-35 a présenté un poids de cent graines moyen de $17 \mathrm{~g}$ à Pobé et $18 \mathrm{~g}$ à Saria. La lignée C2-9-1 a donné un poids de 100 graines supérieur à $20 \mathrm{~g}$ sur les deux sites. L'index de stabilité de la chlorophylle a été plus élevé au niveau de la station de Pobé Mengao. A Saria, les individus présentant les plus faibles scores ont été: C2-111-4 et KVX61-1. IT97K-499-35 a présenté la valeur la plus élevée $(45,05)$. Les individus KVX611, Gorom local et C2-111-4 ont eu les plus faibles valeurs pour le taux de chlorophylle mesuré à Pobé. Sur le site de Pobé Mengao, émergence du Striga gesnerioides a été notée sur les contrôles négatifs C2-111-2 et C2-1114. Tous les autres génotypes ont été résistants à la race 1 de Striga. A Saria, aucune une émergence de Striga gesnerioides a été observée. Sur le site de Pobé Mengao, une émergence du Striga gesnorioides a été notée sur les contrôles négatifs C2-111-2 et C2-1114. Tous les autres génotypes ont été résistants à la race 1 de Striga. A Saria, aucune émergence de Striga gesnerioides a été observée. Les viroses, les taches brunes, et le chancre bactérien ont été observés sur les plantes à Saria. Mais à Pobé Mengao, les génotypes ont été résistants à ces trois maladies. La sénescence a été nulle au niveau des deux localités. Aucun flétrissement de plantes, ni de jaunissent des feuilles n'ont été observé avant la maturité des gousses (Figure 2). Au niveau de l'ACP, la variation totale exprimée par les deux axes a été $76,27 \%$ (Figure 3). L'axe F1 exprimant 49,72\% de la variation totale a été hautement et positivement corrélé avec le rendement et le poids de 100 graines. L'axe 2 exprimant $26,55 \%$ de la variabilité totale a été positivement corrélé avec le nombre de jours à $50 \%$ floraison et $95 \%$ maturité. Il a été négativement corrélé avec l'index de stabilité de la chlorophylle. En relation avec ces deux comportements les individus ont été séparés en fonction de leur rendement et du cycle de semi maturité. Le poids de 100 graines et le rendement ont été positivement corrélés avec les individus C2-9-1, Komcalle et Tiligre. Le nombre de jours à $95 \%$ maturité a été positivement corrélé avec les individus Nafi, Gorom local et KVX 61-1. 
W. J.A. LALSAGA et I. DRABO. /Int. J. Biol. Chem. Sci. 11(6): 2756-2763, 2017

Tableau 1: Moyenne de 50\% floraison, 95\% maturité, poids de 100 graines, de rendement, quantité de chlorophylle pour les génotypes évalués.

\begin{tabular}{|c|c|c|c|c|c|c|c|c|c|c|c|c|}
\hline & \multicolumn{2}{|c|}{$50 \%$ floraison } & \multicolumn{2}{|c|}{ 95\% maturité } & \multicolumn{2}{|c|}{ Poids 100 graines } & \multicolumn{2}{|c|}{ Rendement } & \multicolumn{2}{|c|}{$\begin{array}{c}\text { Émergence du } \\
\text { striga }\end{array}$} & \multicolumn{2}{|c|}{ Taux de chlorophylle } \\
\hline & Pobé & Saria & Pobé & Saria & Pobé & Saria & Pobé & Saria & Po & Saria & Pobé & Saria \\
\hline C2-6-3 & 42,4 & 42,6 & 64,4 & 64,60 & 17,8 & 20,2 & 1187,4 & 1022 & 0 & 0 & 53,89 & 44,52 \\
\hline C2-9-1 & 46 & 45,8 & 66,2 & 66 & 21,4 & 21,8 & 1359,6 & 1501 & 0 & 0 & 56,69 & 43,06 \\
\hline C2-11-3 & 46 & 45,8 & 66 & 66,6 & 17,8 & 20,8 & 1218,8 & 1114 & 0 & 0 & 56,81 & 43,36 \\
\hline C2-18-2 & 41,6 & 40,2 & 64 & 62,4 & 19 & 18,8 & 1000,2 & 1285 & 0 & 0 & 57,32 & 42,08 \\
\hline C2-27-2 & 41,2 & 39,8 & 64 & 61,8 & 19 & 20,2 & 1281,4 & 1504 & 0 & 0 & 56,55 & 42,17 \\
\hline C2-111-2 & 43,4 & 42,6 & 64 & 63,8 & 16 & 18,4 & 1015,6 & 1099 & 1 & 0 & 51,45 & 42,3 \\
\hline C2-111-4 & 48,2 & 46,6 & 68 & 67,2 & 15,4 & 16 & 484,6 & 383 & 1 & 0 & 48,14 & 39,71 \\
\hline Komcalle & 46,2 & 47 & 66,8 & 67,2 & 18,6 & 19,4 & 1218,8 & 1123 & 0 & 0 & 54,54 & 40,44 \\
\hline IT97K-499 & 40,4 & 39 & 63,4 & 60,8 & 17 & 18,2 & 1406,6 & 1386 & 0 & 0 & 57,54 & 45,05 \\
\hline Yiisyande & 40 & 39,4 & 64,8 & 61,2 & 19 & 20,6 & 984,6 & 1285 & 0 & 0 & 51,29 & 40,97 \\
\hline Gorom & 48,6 & 47,8 & 68 & 70,2 & 15 & 16 & 1328 & 1421 & 0 & 0 & 46,57 & 41.96 \\
\hline Nafi & 48 & 45,4 & 67,8 & 67,8 & 18,6 & 19,2 & 953,2 & 1035 & 0 & 0 & 50,29 & 41,28 \\
\hline Niizwe & 38,4 & 37 & 61,6 & 59,8 & 14,4 & 15,4 & 828,2 & 1204 & 0 & 0 & 59,62 & 44,36 \\
\hline Tiligre & 49,2 & 47 & 69 & 66,8 & 19,2 & 20,8 & 1390,4 & 1226 & 0 & 0 & 55,06 & 43,73 \\
\hline KVX-61-1 & 48,6 & 48,2 & 67,6 & 70,8 & 13,8 & 15,2 & 1312,6 & 1340 & 0 & 0 & 48,07 & 39,89 \\
\hline Moyenne & 44,54 & 43,61 & 65,70 & 65,1 & 17,46 & 18,73 & 1131,3 & 1195,2 & & & 53,59 & 42,32 \\
\hline $\mathrm{CV} \%$ & 4,2 & 4,3 & 2,3 & 3,6 & 4,3 & 9,1 & 27,3 & 29,6 & & & 4,6 & 8,0 \\
\hline PPDS & 2,284 & 2,249 & 1,870 & 2,924 & 0,9623 & 2,115 & 391,7 & $449,80,009$ & & & 3,158 & 4,245 \\
\hline Probabilité & $<.001$ & $<.001$ & $<.001$ & $<.001$ & $<.001$ & $<.001$ & 0,008 & & & & $<.001$ & 0,438 \\
\hline
\end{tabular}




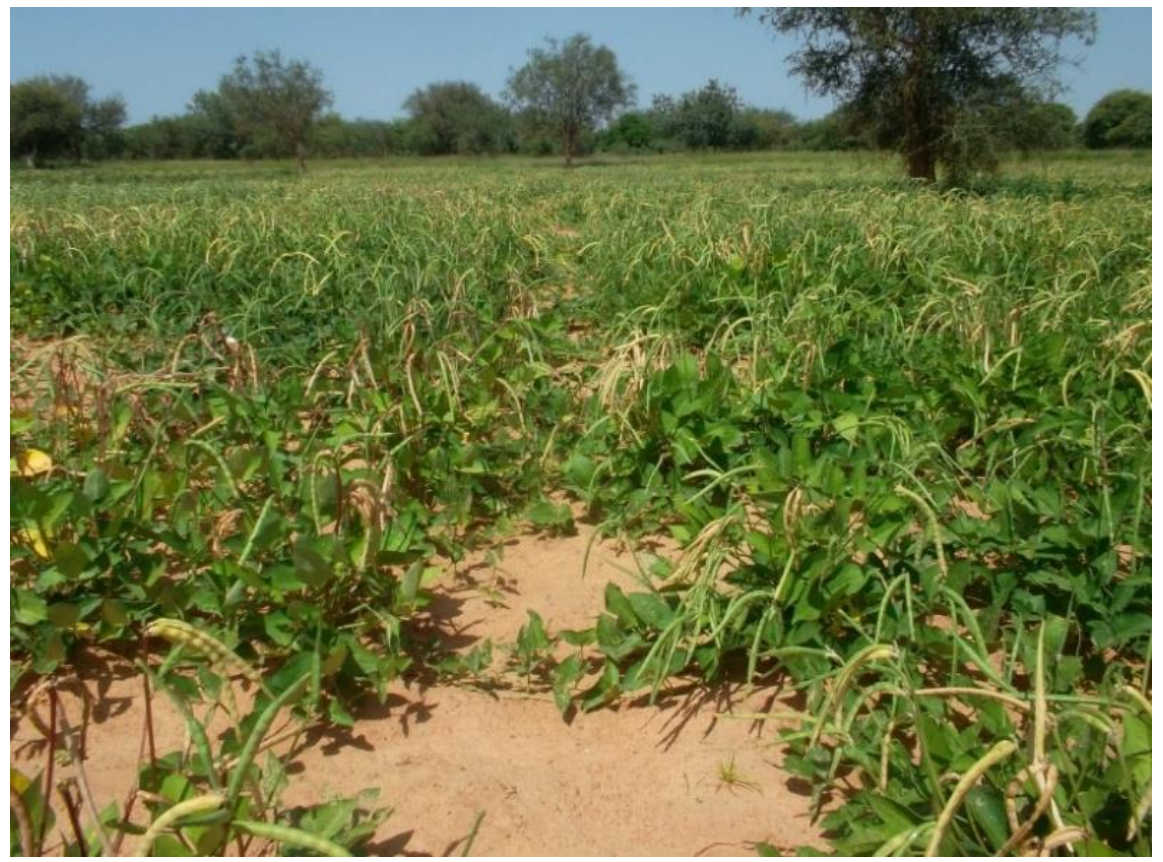

Figure 2: Plantes C2 et variétés au stade de maturité sur le site de Pobe Mengao.

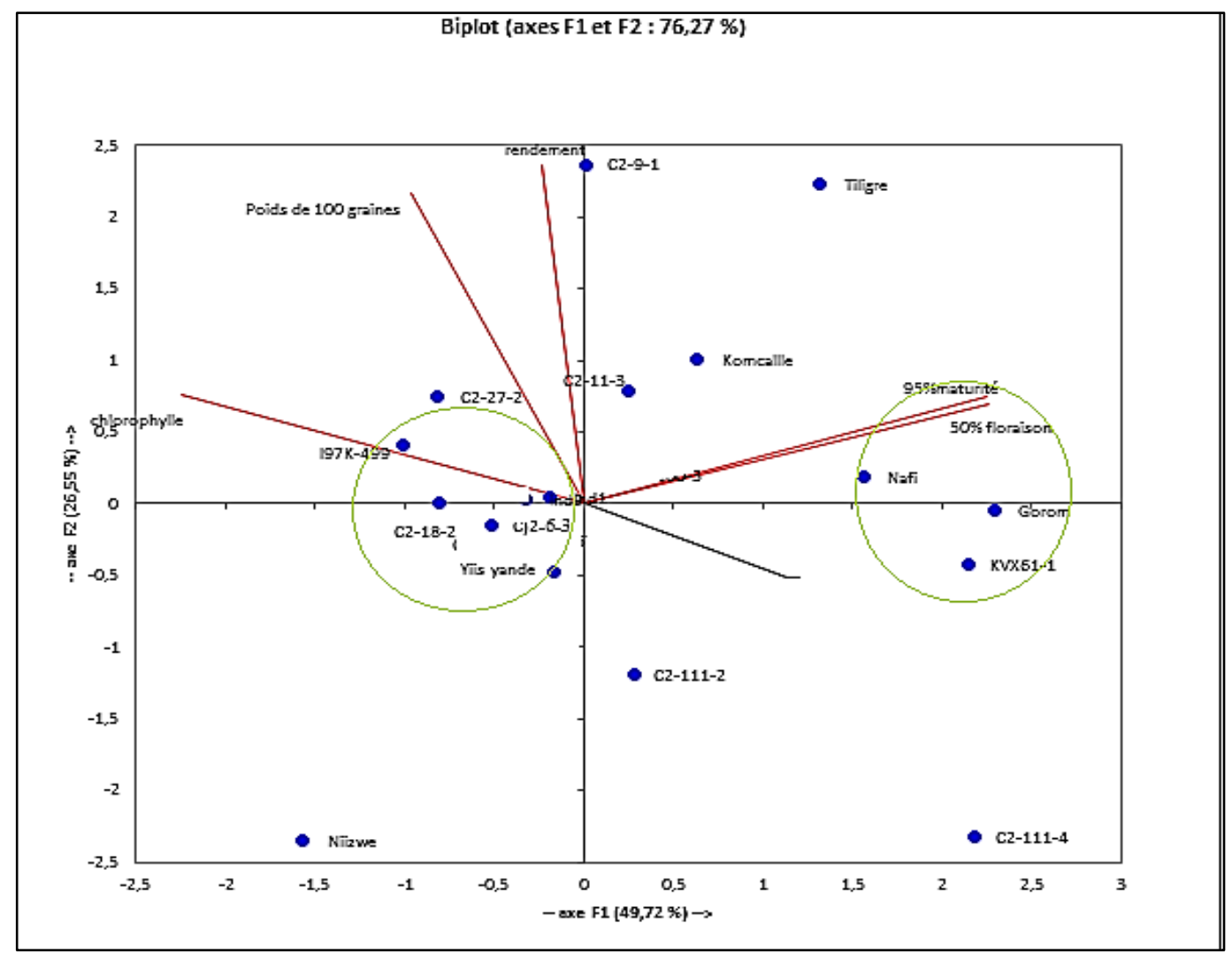

Figure 3: Représentation des individus dans le plan des axe 1-2 de l'ACP. 


\section{DISCUSSION}

Les expérimentations ont eu lieu dans des conditions de variation de la pluviométrie d'une région à l'autre. La station de Saria a reçu 870,6 mm d'eau et Pobé Mengao 512 $\mathrm{mm}$. Une telle évaluation du matériel de production permet de choisir des lignées qui seront vulgarisées en milieu paysan. En général, les variétés ont fleuri et atteint la maturité plus vite à Saria qui représente le lieu où il $\mathrm{y} a$ eu le maximum d'humidité. La faible pluviométrie enregistrée à Pobé Mengao a eu un effet sur les génotypes, empêchant les variétés d'exprimer tout leur potentiel. Belko (2013) et Ishiaku et Aliyu (2013) ont constaté que les plantes fleurissent et atteignent la maturité plus vite dans les conditions de maximum d'humidité. Il y a eu une variation au niveau du rendement grain entre les deux localités. Les plantes ont produit plus sur le site qui a reçu la plus grande quantité d'eau. Cette différence peut s'expliquer par l'insuffisance de la quantité d'eau appliquée ou l'influence des facteurs climatiques notamment les fortes températures enregistrée à Pobe Mengao. Alidu et al.,(2013) ont trouvé que le stress hydrique réduit considérablement le rendement grain. La mauvaise répartition spatiale et temporelle de la pluviométrie ainsi que les températures élevées accentuent la sécheresse qui entrave la production du niébé (Kihindo et al., 2015). Les résultats montrent que les lignées: C2-6-3, C2-9-1, C2-11-3, C2-18-2 et C2-27-2 produisent assez bien en condition de stress que de non stress. Compte tenu de leur capacité à produire plus de 1200 $\mathrm{kg}$ dans les deux localités, les génotypes C2-9-1 et C2-27-2 peuvent être recommandés aux producteurs. Gbaguidi et al., (2015) rapportent qu'une caractérisation est nécessaire pour une utilisation rationnelle des ressources. Au plan général des performances agronomiques, les lignées se sont aussi bien comportées, voire mieux comportées que les variétés vulgarisées. L'augmentation de la teneur en chlorophylle totale chez toutes les variétés sous la restriction hydrique au stade végétatif et à la floraison révèle que le stress hydrique provoqué par une baisse de la quantité d'eau disponible entraîne une concentration du pigment photosynthétique chez le niébé. Nana et al. (2009) ont souligné une diminution du contenu foliaire en chlorophylle chez le gombo lorsque les plants subissent un stress hydrique et stress hydrique appliqué serait modéré pour les plants de gombo. Le regroupement de l'ACP réalisé avec le nombre de jours de $95 \%$ maturité, le poids de 100 graines, le taux de chlorophylle et le rendement grain obtenu sur le site de Pobé Mengao a permi d'identifier deux groupes. Le groupe 1 renferme des individus précoces à rendement modéré qui sont: C211-3, Komcalle, Nafi et Gorom local. Le deuxième groupe est formé par des individus extra précoces à rendement modéré: C2-6-3, IT97K-499-35, C2-18-2 et C2-27-2.

\section{Conclusion}

L'évaluation des lignées issues de la sélection récurrente a mis en exergue une conservation du rendement, de la précocité et une amélioration du poids de 100 graines par rapport aux deux parents du croisement. Toutes les lignées ont atteint $95 \%$ maturité avant le 71eme jour. Elles sont aussi résistantes à la race 1 du Striga. Parmi ces différents résultats de progrès génétique, quelques lignées se distinguent des autres. Il ressort que la lignée C2-9-1 présente le meilleur rendement, un poids de 100 graines supérieur à $21 \mathrm{~g}$ et des graines blanches. Ce génotype pourrait par conséquent être recommandé aux producteurs.

\section{CONFLIT D'INTERETS}

Les auteurs attestent qu'il n'y a aucun conflit d'intérêts.

\section{CONTRIBUTIONS DES AUTEURS}

WJAL a collecté, analysé les données et a rédigé le papier. ID a participé la correction du manuscrit.

\section{REFERENCES}

Alidu MS, Atokple IDK, Akromah R. 2013. Genetic analysis of vegetative-stage drought tolerance in cowpea. Greener 
Journal of Agricultural Sciences, pp. 481-496.

DOI:

http://www.gjournals.org/GJAS/GJAS\% 20Pdf/2013/June/030613516\%20

Belko N. 2013. Phenotypage pour la tolérance à la sécheresse de fin-de-cycle chez le niébé (vigna unguiculata (1.) walp.) thèse de doct. univ de ouaga, $100 \mathrm{p}$.

Chiulele R. 2010. Breeding cowpea (Vigna unguiculata (L) Walp.) for improved drought tolerance in Mozambique. These de doct. University of kwazulu-Natal, $156 \mathrm{p}$.

Fatokun C, Boukar O, Muranaka S, Chikoye D. 2009. Enhancing drought tolerance in cowpea. African Crop Science Society, 9: 531-536. DOI: http://www .cabdirect.org/abstracts/ 20133232420.html

Gbaguidi A, Assogba P, Dansi M, Yedomonhan H, Dansi A. 2015. Caractérisation agromorphologique des variétés de niébé cultivées au Bénin. Int. J. Biol. Chem. Sci., 9(2): 1050-1066, DOI: http://dx.doi.org/10.4314/ijbcs.v9i2.40.

Hall E. 2004. Breeding for adaptation to drought and heat in cowpea. Europ $J$. Agronomy, 21: 447-454. DOI:10.1016/j.eja.2004.07.005.

Ishiyaku MF, Aliyu H. 2013. Field evaluation of cowpea genotypes for drought tolerance and Striga resistance in the dry savanna of north-west Nigeria. International Journal of Plant Breeding and Genetics, 7(1): 47-56, DOI: 10.3923/ijpbg.2013.47.56.

Issoufou $\mathrm{H}$, Boubacar $\mathrm{S}$, Adam T, Boubacar Y. 2017. Identification des insectes, parasites et évaluation économique de leurs pertes en graines sur les variétés améliorées et locale de niébé en milieu paysan à Karma (Niger) Int. J. Biol. Chem. Sci., 11(2): 694-706. DOI: https://dx.doi.org/10.4314/ijbcs.v11i2.13

Kihindo AP, Bazie RH, Ouedraogo RF, Some PP, Zombre G, Tozo K. 2015. Effets de la date de semis et du régime hydrique sur la réponse agro morphologique de deux variétés de niébé (KN1 ET KVX 61-1) au Burkina Faso international. Journal of Innovation and Applied Studies, 564-573. DOI: https://www.researchgate.net/publicatio $n / 296431747$

Muchero W, Ehlers JD, Roberts PA. 2008. Seedling stage drought-induced phenotypes and drought-responsive genes in diverse cowpea genotypes. Crop Sci., 48: 541-552. DOI: 10.2135/cropsci2007.07.0397.

Nana R, Tamini Z, Sawadogo M. 2009. Effets d'un stress hydrique intervenu pendant le stade végétatif et la phase de floraison chez le gombo Int. J. Biol. Chem. Sci. 3(5): $\quad$ 1161-1170. DOI: https://www.ajol.info/index.php/ijbcs/art icle/viewFile/51094/39776

Rabe M, Baoua I, Adeoti R, Sitou L, Amadou L, Pittendrigh B, Mahamane S. 2017. Les déterminants socioéconomiques de l'adoption des technologies améliorées de production du niébé diffusées par les champs écoles paysans dans les régions de Maradi et Zinder au Niger. Int. J. Biol. Chem. Sci., 11(2): 744-756, DOI: https://dx.doi.org/10.4314/ijbcs.v11i2.17

Tignegré JB. 2010. Genetic study of cowpea (Vigna unguiculata(l.) Walp.) resistance to Striga gesnerioides(willd.) vatke in Burkina Faso. Ph.D. Dissertation. Univ. of kwazulu-Natal, $170 \mathrm{p}$. 